Objectives: To explore the effect of nicotine on matrix metalloproteinases (MMPs) and RANKL expression from RA-FLS and its possible intracellular signalling mechanism.

Methods: Synovial tissues were obtained from 45 patients with active RA as well as 11 osteoarthritis $(\mathrm{OA})$ and 11 noninflammatory orthopaedic arthropathies (Orth.A) patients for control. The expression of AChR $\alpha 7$ in synovial membrane and cultured FLS were detected by immunohistochemistry staining and Western blot. RA-FLS were treated in vitro with different concentration of nicotine and its effect on RA-FLS viability was evaluated by cell counting kit-8. After nicotine pretreatment on TNF- $\alpha$ stimulated RA-FLS, the expression of MMP-2, MMP-3, MMP9, TIMP-1, TIMP-2, RANKL, and OPG in culture supernatant were measured by ELISA, while the change of AP-1 pathway including c-Fos and c-Jun were detected by quantitative real-time PCR and Western blot.

Results: Immunohistochemical analyses showed intense endochylema staining for $\mathrm{AChR} \alpha 7$ mainly in lining layer. The percentage of both lining and sublining $A C h R \alpha 7$ positive cells were significantly higher in RA than that in OA or Orth.A (figure 1A). Further western blot showed significantly higher expression of $A C h R \alpha 7$ in RA-FLS than that in Orth.A-FLS $(p=0.003$, figure 1B). Nicotine $(0.1 \mu \mathrm{M} \sim 50 \mu \mathrm{M})$ showed no cytotoxicity on RA-FLS proliferation. Pretreatment with $50 \mu \mathrm{M}$ nicotine for 24 hours significantly promoted the secretion of MMP-3 and RANKL but inhibited TIMP-1 secretion in TNF- $\alpha$ stimulated RA-FLS (all $\mathrm{p}<0.05$, figure $1 \mathrm{C}$ ). Furtherly, $25 \mu \mathrm{M}$ and $50 \mu \mathrm{M}$ nicotine treatment for 24 hours upregulated both mRNA and protein expression of c-Fos but not c-Jun which indicated that nicotine might activate AP- 1 signalling pathway by $c-F o s$ (all $p<0.05$, figure 1D).

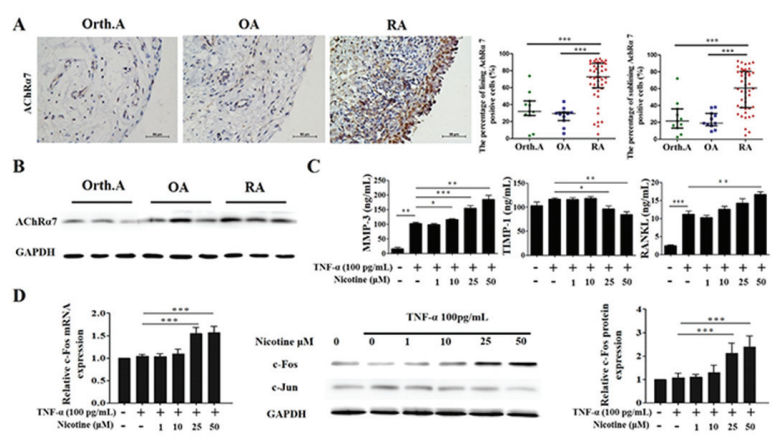

Abstract SAT0036 - Figure 1. Effects of nicotine on the secretion of MMPs, RANKL and OPG as well as AP-1 signalling pathway activation in RA-FLS. A Immunohistochemistry staining for $A C h R \alpha 7$ in RA synovium. The data were representative as median and interquartile range from $45 \mathrm{RA}, 11 \mathrm{OA}$ and 11 Orth. A patients. B The expression of AChR $\alpha 7$ in RA-FLS was measured by Western blot. C After nicotine treatment for 24 hours, the levels of MMP-3, TIMP-1 and RANKL in culture supernatant of TNF- $\alpha$ stimulated RAFLS were measured by ELISA. D After nicotine treatment for 24 hours, the expression of CFos and c-Jun in RA-FLS was measured by quantitative real-time PCR and Western blot. The data were representative as means \pm SD from six different RA patients, ${ }^{*} P<0.05$, ${ }^{\star *} P<0.01,{ }^{* \star *} P<0.001$

Conclusions: Nicotine can promote MMP-3 and RANKL expression through overexpressed $A C h R \alpha 7$ in RA-FLS which might be involved in the pathogenesis of osteogenesis and bone destruction in RA.

Acknowledgements: This work was supported by National Natural Science Foundation of China (no. 81471597 and 81671612), Guangdong Natural Science Foundation (no. 2017A030313576 and 2017A030310236) and Fundamental Research Funds for the Central Universities (no. 17ykjc12).

Disclosure of Interest: None declared

DOI: 10.1136/annrheumdis-2018-eular.5115

\section{SAT0037 IGURATIMOD AMELIORATES BLEOMYCIN-INDUCED ALVEOLAR INFLAMMATION AND PULMONARY FIBROSIS IN MICE BY SUPPRESSING EXPRESSION OF MMP-9}

L. Zhao, B. Mu, R. Zhou, C. Huang. Department of Rheumatology, Beijing Hospital, National Center of Gerontology, Beijing, China

Background: Interstitial lung disease (ILD) occurs in 15\% of patients with connective tissue diseases (CTD) It contributes significantly to morbidity and mortality, and is difficult to manage ${ }^{(1)}$. Despite advances in management strategies, CTD-ILD have a significant adverse effect on quality of life and is still a leading cause of mortality, highlighting an urgent need for optimal treatment regimens. It is known that inflammatory cytokines played an important role in alveolitis and the development of pulmonary fibrosis ${ }^{(2)}$. Iguratimod (IGU) has a reported effect on preventing the inflammatory processes by inhibiting the production of various inflammatory cytokines ${ }^{(3)}$

Objectives: To investigate the potential therapeutic efficacy of IGU on mouse model of bleomycin (BLM) induced pulmonary fibrosis.

Methods: A total of $75 \mathrm{C} 57 \mathrm{BL} / 6$ mice were randomly and evenly divided into con trol group, BLM (5 mg/kg) group, BLM +IGU $(90 \mathrm{mg} / \mathrm{kg})$ group BLM +methylprednisolone (MP, $10 \mathrm{mg} / \mathrm{kg}$ ) group and BLM +pirfenidone (PF $100 \mathrm{mg} / \mathrm{kg}$ ) group. The mice were sacrificed on day 7 , day 14, day 28 respectively. The lung tissue was examined by Hematoxylin and eosin staining and Masson staining to evaluate the degree of alveolitis and fibrosis, and plasma cytokines were measured.

Results: Histopathological results showed that IGU attenuated BLM-induced alveolar inflammation and decreased collagen deposition in lung tissue from day 7 till day 28. Both the pathological alveolitis scores and fibrosis scores in drug treated group (IGU group, MP group and PF group) were decreased dramatically compared with BLM group on day 7 , day 14 and day 28 respectively $(p<0.05)$. There were no statistical significances among those three groups. Cytokine profile showed that IGU decreased the level of tumour necrosis factor- $\alpha$ (TNF- $\alpha$ ), interleukin (IL)-1, IL-6 and matrix metalloproteinase (MMP) - 9 which were up-regu lated by BLM on day 7 , day 14 and day 28 respectively $(p<0.05)$. Furthermore, there is a strong correlation between the severity of the pulmonary fibrosis and the plasma MMP-9 levels $(\longleftrightarrow \longleftrightarrow=0.836, p<0.001)$.

Conclusions: IGU was effective in suppressing BLM-induced alveolar inflammation and pulmonary fibrosis, and it has the equal anti-inflammatory and anti-fibrotic effects as MP or PF. The therapeutic effects by IGU may be attributed to the inhibition of MMP-9 either directly or indirectly. Therefore, IGU presents as a promising agent for pulmonary fibrosis treatment.

\section{REFERENCES :}

[1] Demoruelle MK, Mittoo S, Solomon JJ. Connective tissue disease-related interstitial lung disease. Best practice \& research Clinical rheumatology 2016;30:39-52.

[2] Luzina IG, Todd NW, Sundararajan S, Atamas SP. The cytokines of pulmonary fibrosis: Much learned, much more to learn. Cytokine 2015;74:88100.

[3] Mucke HA. Iguratimod: a new disease-modifying antirheumatic drug. Drugs of today $2012 ; 48: 577-86$.

Disclosure of Interest: None declared

DOI: 10.1136/annrheumdis-2018-eular.2289

\section{SAT0038 MEMBRANE-ASSOCIATED AMPD2, A NOVEL REGULATOR IN SHIFTING THE BALANCE BETWEEN EXTRACELLULAR ATP AND ADENOSINE?}

L. Ehlers, A. Kuppe, A. Damerau, Y. Chen, F. Buttgereit, T. Gaber. Department of Rheumatology and Clinical Immunology, Charité - Universitä Tsmedizin Berlin, Berlin, Germany

Background: Extracellular ATP and adenosine are potent immunomodulatory molecules that accumulate in states of inflammation. ATP/ADP are released from damaged or stressed cells and sequentially catabolized to AMP which is then catabolized to adenosine by the action of the ectonucleotidases CD39 and CD73, which drives a shift from an ATP-driven proinflammatory environment to an antiinflammatory milieu induced by adenosine. Intracellularly, AMPD2 which encodes one of three known AMP deaminase homologs executes AMP deamination to IMP thereby reducing the formation of adenosine. Here, we postulate that this mode of action is also present on the cell surface of immune cells, which may lead to an increased state of inflammation such as found in chronic inflammatory diseases.

Objectives: Therefore, we analysed surface AMPD2 expression and its modulation on distinct human cell lines and primary human immune cells.

Methods: To this end, surface AMPD2 expression was evaluated on cell lines (THP1, Jurkat, HMEC1, and HEK293), human PBMCs and isolated monocytes by flow cytometry. Moreover, co-expression of surface AMPD2, CD73 and CD39 has been analysed on PBMCs and isolated monocytes. Association of surface AMPD2 and cell death was visualised using annexin $\mathrm{V}$ and 7-AAD staining and examined by flow cytometry. In addition, expression of AMPD2 was analysed by immunoblot of precipitated AMPD2 from membrane fractions and by mass spectrometry after precipitation from membrane fractions and from biotinylated surface-molecules using the surface AMPD2 positive cell line HEK293.

Results: Here, we demonstrate that (i) surface AMPD2 is present on T cells and monocytes in PBMCs from healthy donors, (ii) that LPS enhances surface expres sion of AMPD2 in PBMCs on both cell populations after 24 hour, (iii) that LPS also enhances surface expression of AMPD2 in isolated monocytes, (iv) that surface AMPD2 does not correlate to the expression of the ectonucleotidases CD39 and CD73 and (v) that all cell lines analysed are capable of expressing surface 